DR. J. H. MASon KNox, JR., Baltimore: The institutional care of young babies is a question of the characier both of the institution and of the foster home. In institutions as constructed years ago and which have an inadequate number of poorly trained attendants, the infant mortality is frightfully high; much higher than in even indifferent foster homes.

The term "mothering" has been objected to on the ground that it may imply too much fondling. We might perhaps better substitute the phrase, "individualizing care." I agrce with Dr. Hess that too much unintelligent mothering is a great disadvantage; but 1 believe that the baby, absolutely dependent, as it is, on others, does best when it has individualizing care; this cannot be obtained unless there is one caretaker for every three or four children. When this is furnished so that the babies' wants are promptly supplied, and there is abundant air space and quiet, it really does not make much difference whether the baby is in an institution or in a foster home.

\section{SIMPLIFIED TECHNIC IN LAMINECTOMY *}

WITH DESCRIPTION OF COMBINED LAMINECTOMY AND SPINE FIXATION BY BONE TRANSPLANT

\section{F. J. GAENSLEN, M.D. MILWAUKEE, WIS.}

The operation of laminectomy has become so well standardized that one feels a certain hesitancy in presenting modifications. However, the technic that I have employed during the past few years has been found rather simpler than that of methods previously described, so that a description of it appears warranted.

Briefly, the essential point lies in the fact that instead of stripping the soft parts and the periosteum from the posterior vertebral spines on either side and then cutting off the spines at their bases, the spines are split longitudinally into right and left halves, just as in the Albee operation for insertion of a bone transplant in Pott's disease. However, instead of deflecting the halves on one side only, as in the Albee technic, the split halves are deflected on both sides. It is then a very simple matter to separate the periosteum with attached soft parts from the laminae as far laterally as the articular processes. The posterior spines should be split well down to their bases. There is then left only a small remnant of the process to be cut away with bone cutting forceps. The cutting away of the laminae can be accomplished by any one of the numerous methods advised.

Details of Technic.-The first steps are like those in the Albee spine fixation. A curved incision is made slightly to one side of the row of posterior spinous processes through the skin and subcutaneous tissue. The flap is rapidly reflected, exposing the deep fascia on either side of the middle line. With a stout knife, a straight incision is made down the middle line, splitting the supraspinous ligament and incising or at least marking the tips of the spinous processes themselves. A tenotomy knife is now used to dip down betwcen the individual spinous processes, dividing the interspinous ligaments. With a very thin chisel the spines are now split through the line indicated, care being taken to keep as close to the middle line as possible. On reaching the base of the spinous processes, the chisel is driven slightly to one side, and the corresponding halves are broken and deflected to that side. With a gauze sponge over a periosteal elevator, the

* Read before the Section on Orthopedic Surgery at the Sixty-Eighth Annual Session of the American Medical Association, New York, June, 1917.

periosteum is now stripped from the corresponding laminae to the articular processes. This is easily and quickly accomplished without injury or fraying of the periosteum with the clean start obtained, as just indicated. It is the ease and rapidity with which this can be done, and the relatively slight hemorrhage encountered as compared with other methods, which particularly recommends this procedure. With a few strokes of the chisel the opposite standing halves of the posterior spines are now broken off close to their bases, and deflected to the side, the periosteum being stripped back as on the opposite side. The next step consists in biting off with suitable forceps the remaining portion of the bases of the spinous processes and cutting through the laminae by any one of a number of suitable methods. The Hudson drill and a number of rongeur forceps of suitable size and shape will answer the purpose very well. After removal of the laminae, the spinal contents are dealt with as circumstances demand.

The closure of the wound is in no way complicated by the method of approach outlined. In fact, the clean cut wound edges showing the split halves of the spinous p:ocesses and the split supraspinous and interspinous ligaments offer good opportunity for accurate approximation. When the split spines are not too thick a stout,
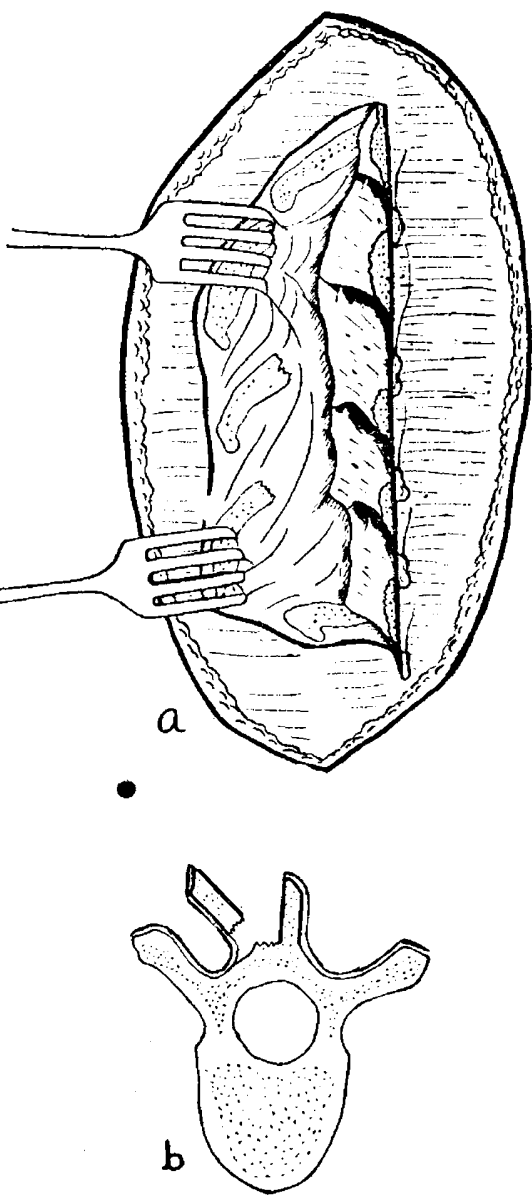

Fig. 1.-Posterior spinous processes (a) split in the middle; the left halves are denuded laminae on that side exposed the denuded laminae on that side exposed; the
right halves remain standing; $b$ cross section, showing splitting of posterior spinous process and denudation of lamina on left. kidney infection and trophic ulcers. The laminectomy wound had healed perfectly and the split halves of the 
spinous processes were firmly adherent to one another, though with a little force they could be separated with
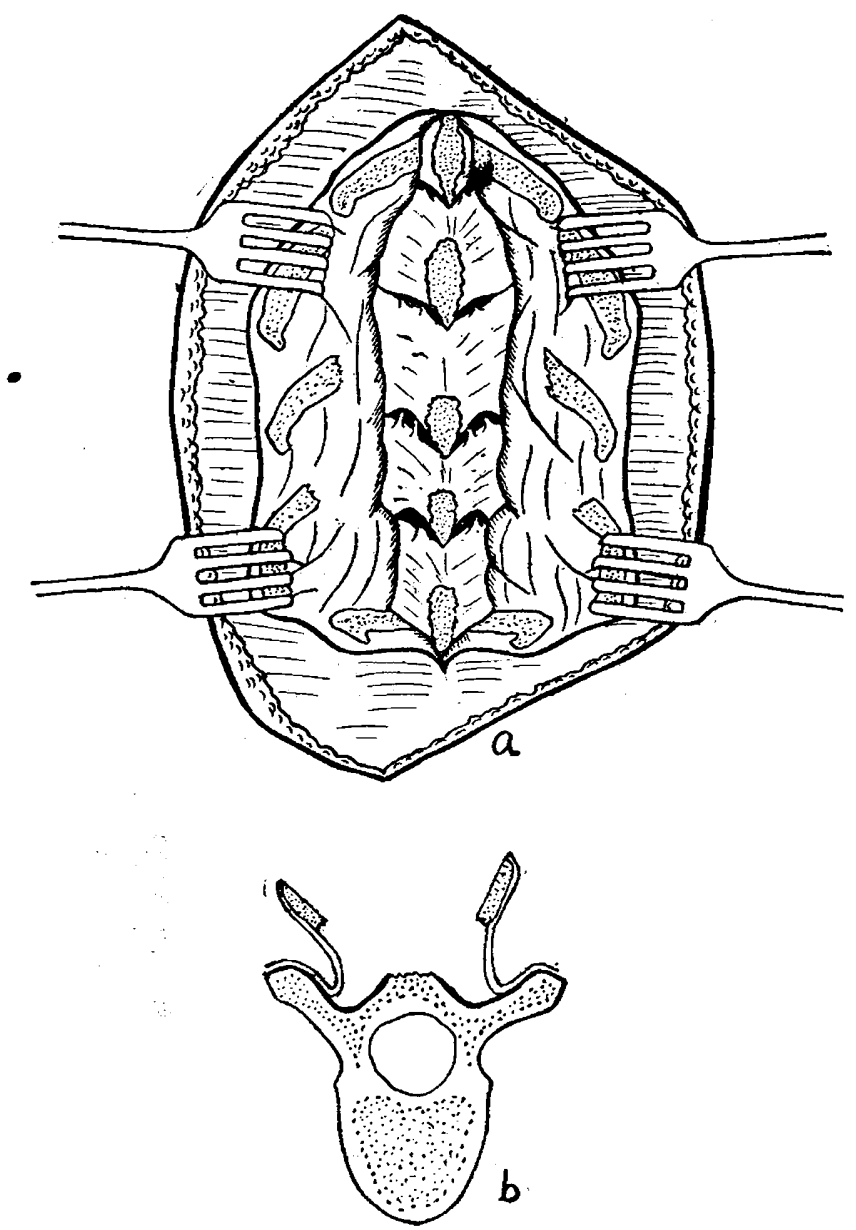

Fig. 2.-Broken spinous processes (a) deflected on both sides, exposing denuded laminae on both right and left; $b$, cross section.

a snap, showing that bony union was well under way.

The advantages of the method of approach, outlined above, are: (1) rapidily; (2) diminished trauma; (3) diminished hemorrhage, and (4) more accurate and firmer closure, by virtue of the union of the bony components.

In certain instances, after laminectomy for urgent cord pressure symptoms, it may be desirable to fix the spine by means of a tibial transplant immediately after decompression of the cord. After the usual operation practiced heretofore, in which the spinous processes are sacrificed, a bridging over of the laminectomized area by means of a tibial graft would be a hazardous proceeding, the graft receiving bony contact only above and below the laminectomized region. This hazard would naturally increase with the length of the laminectomized area It may be regarded as a universally accepted fact that a bone graft has far better chances of becoming firmly united if it is in contact with numerous healthy bone surfaces. The method of approach, indicated above, by preserving the spinous processes and therefore retaining the usual number of bony contact points, paves the way for a successful combination of laminectomy and spine fixation by means of the bone graft. This plan was carried out in two cases, in both of which a diagnosis of vertebral tuberculosis had been made with urgent cord symp- toms. After decompression of the cord, by laminectomy, performed according to the plan just outlined, several healthy spines above and below were split after the usual manner. The left halves were deflected while the right halves remained standing. A graft of sufficient length was then cut and put in place, bridging over the laminectomized area and finding anchorage above and below on the standing halves.

In both instances, primary union was obtained. In the first case, one with a middorsal lesion, the spine is held perfectly rigid, the graft fulfilling its mechanical function perfectly. In the second, a case of cervical Pott's disease with urgent symptoms, death occurred about four weeks after operation from an ascending urinary infection.

To be sure, indications calling for the employment of this combination of procedures will be encountered only rarely. Traction and recumbency in the hands of most orthopedic surgeons will prove adequate in the vast majority of cases, but in certain cases of Pott's disease, possibly also in some cases of fracture of the spine, a combination of the two will be found desirable. The experience in the cases just reported would indicate also that the method is feasible.

141 Wisconsin Street.

\section{ABSTRACT OF DISCUSSION}

Dr. Edwin W. Ryerson, Chicago: My experience with this operation is confined to one case, which I had recently, for fracture-dislocation of the spine. The procedure of $\mathrm{Dr}$. Gaenslen was not exactly followed, in that I used a motor saw to split the spinous process, thereby causing less traumatism than when the chisel and mallet are used. The operation was nearly bloodless, much less hemorrhage being encountered than when the spinous processes are exposed and cut away by the rongeur. This procedure is the more valuable, as it gives an opportunity to put in a bone splint in cases of pressure on the spine caused by tuberculosis. These cases do not often require laminectomy; but when they
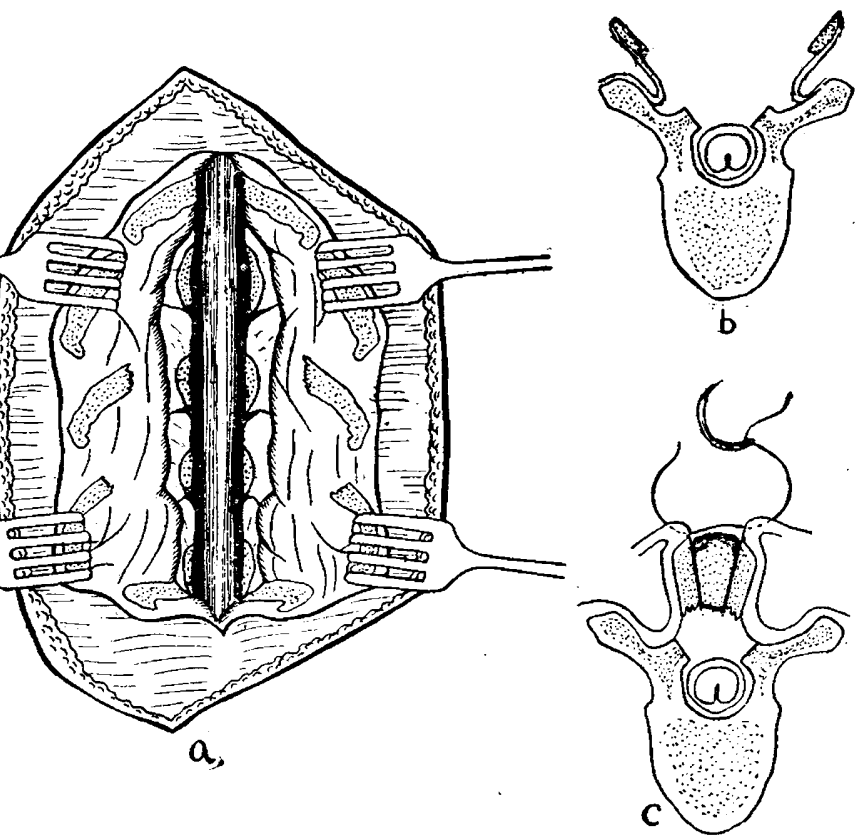

Fig. 3.-Laminąe removed $(a)$; cord exposed; $b$, cross section; $c$ cross section of vertebra in laminectomized area, showing bone graft in place and suture introduced.

do, one would hesitate to do a simple decompressive laminectomy, if there were no possibility of fixing the spine afterwards; but with one of the tibial splints, we may properly hesitate less to do it than in the past. 